\title{
ON ADAPTIVITY FOR DIFFUSION PROBLEMS USING TRIANGULAR ELEMENTS *
}

\author{
Claudio Padra and Marcelo J. Vénere \\ División Mecánica Computacional, DIA \\ Centro Atómico Bariloche, CNEA \\ 8400 San Carlos de Bariloche - Argentina
}

- This work was partially supported by Consejo Nacional de Investigaciones Científicas y Técnicas (CONICE'I), under PID-BID No. 40/92

\begin{abstract}
In this work an adaptive scheme to solve diffusion problems using linear and quadratic triangles is presented. The densification algorithm, based on the subdivision of the selected elements, and the error estimator used are first described. We pay special attention to the behavior of the estimator. It has two contributions: the residual term and the fiux-jump term. Babuska and co-workers have shown that for bilinear quadrilaterals, the first term is negligible, but for biquadratic, it is the dominant term. We show evidence suggesting that these results can not be extended to triangular elements when the problem has a singular solution. We found in this case that if the flux-jump term is neglected, the expected rate of convergence can not be obtained. Finally, some remarks about the whole adaptive process are discussed.
\end{abstract}

\section{INTRODUCTION}

In the numerical approximation of partial differential equations, one often encounters the problem that the overall accuracy of the numerical solution is degraded by a non-uniform distribution of the error, specially when the solution of the continuous problem has local singularities. The need for accurate solutions has made the use of adaptive procedures very attractive and necessary for large scale problems. This procedure involve two steps: the evaluation of an error estimate and local error indicators (i.e.: estimates per element), and the mesh refinement process based upon these indicators.

For the mesh refinement process we use an algorithm based in the subdivision of simplices which is briefly described in section 3 . This idea was successfully used by several authors (see for example [6] or [7]) and is specially attractive for adaptive process because it is possible to guarantee that elements will not degenerate.

In section 4 we describe a posteriori error estimator for diffusion problems, based on the computation of the residual and flux jump of the approximate solution. Babuska and Miller [2] studied the contributions of the two error terms for linear elliptic systems. They showed that for a mesh of rectangular elements the residual term can be neglected when estimating errors of bilinear approximations, and Babuska and Yu [3] showed that if the solution is locally sufficiently smooth 
then the boundary term can be neglected when estimating errors of biquadratic approximations. From the implementation point of view, the flux-jump term has more technical difficulties than the residual term, and these difficulties increase when working in three dimensional problems, therefore the idea of dropping that term results very attractive. It is natural to ask whether in a triangular mesh the previous results hold. In this paper we study the behavior of the error estimator and error indicator for different type of problems and the possibility that we can remove one of the error terms for linear or quadratic approximations. The results are presented in section 5.

Finally, in section 6 the behavior of the whole adaptive process is discussed.

\section{THE MODEL PROBLEM}

Let $\Omega$ be a bounded polygon in $\Re^{2}$. We consider elliptic problems:

$$
\begin{aligned}
-\operatorname{div}(k \nabla u)+b u & =f \text { on } \Omega \\
u & =\delta \text { in } \partial \Omega_{1} \\
a \frac{\partial u}{\partial n} & =\gamma \text { in } \partial \Omega_{2} \\
a \frac{\partial u}{\partial n}+\alpha u & =\beta \text { in } \partial \Omega_{3}
\end{aligned}
$$

where $k$ is a Lipschitz function such that $k(x) \geq k_{0}>0$ and $b(x) \geq 0$ are bounded functions . The boundary of $\Omega$ is the disjoint union of $\partial \Omega_{i}$. Let us denote:

$$
a(u, v)=\int_{\Omega} k \nabla u \nabla v+b u v+\int_{\partial \Omega_{3}} \alpha u v
$$

and

$$
F(v)=\int_{\Omega} f v+\int_{\partial \Omega_{2}} \gamma v+\int_{\partial \Omega_{3}} \beta v
$$

the bilinear and linear form associated to 1 . We use the standard notation for Sobolev spaces, $H^{s}(\Omega)$ for $s \geq 0$ and $H_{0}^{1}(\Omega)$; and the usual norms and seminorms \|\|$_{s, \Omega} .||_{s, \Omega}$. We introduce, as usual $V=\left\{u \in H^{1}(\Omega):\left.u\right|_{\partial \Omega_{1}}=\delta\right\}$ and $V_{0}=\left\{u \in H^{1}(\Omega):\left.u\right|_{\partial \Omega_{1}}=0\right\}$. Hence $a($,$) is V_{0}$-elliptic. The weak formulation of problem 1 may be written in the form:

$$
\begin{array}{cc}
\text { Given } f \in H^{-1}(\Omega), \delta \in & H^{\frac{1}{2}}\left(\partial \Omega_{1}\right), \gamma \in H^{-\frac{1}{2}}\left(\partial \Omega_{2}\right), \beta \in H^{-\frac{1}{2}}\left(\partial \Omega_{3}\right) \\
\text { find } u \in V \text { such that }: & a(u, v)=F(v) \forall v \in V_{0}
\end{array}
$$

Let $\mathcal{T}_{h}$ be a regular family of triangulation of $\Omega$, i.e. there exists a constant $\sigma$ such that $h_{K} \leq \sigma \rho_{K}$ where, as usual, $h_{K}$ denotes the diameter of $\mathrm{K}, \rho_{K}$ the supremum of the diameters of the spheres inscribed in $K$ and $h$ the maximum of $h_{K}$. 
Let $X_{h}=\left\{v \in H^{1}(\Omega):\left.v\right|_{T} \in \mathcal{P}_{k}, \forall T \in \mathcal{T}_{h}\right\}$ be the finite dimensional space of continuous functions where $\mathcal{P}_{k}$ denotes the set of polynomials of degree not greater than $k$. We shall assume that $\delta \in \Phi_{0}\left(X_{h}\right)$ where $\Phi_{0}$ is the trace operator on $\partial \Omega_{1}$, i.e. $\Phi_{0}(u)=\left.u\right|_{\partial \Omega_{1}}$. Now, we can sel $V_{h}=X_{h} \cap V$ and $V_{h_{0}}=X_{h} \cap V_{0}$. The finite element solution $u_{h} \in V_{h}$ is defined by:

$$
a\left(u_{h}, v\right)=F(v) \quad \forall v \in V_{h_{0}}
$$

Let $e=u-u_{h}$ denote the error of this approximation. It is well-know [4] that if $u \in H^{k+1}(\Omega)$ then :

$$
|e|_{1, \Omega} \leq C h^{k}|u|_{k+1, \Omega}
$$

\section{THE DENSIFICATION ALGORITHM}

The densification algorithm we use is based in the idea proposed in [7]. Basically it has two steps, subdivision of the selected elements (triangles in 2D, tetraedral in $3 \mathrm{D}$ ), and the conforming process.

For the two dimensional case, the algorithm is:

Each triangle with the error indicator $\eta_{T}$ greater than a certain cote $\eta_{m}$ is subdivided in four similar triangles (see figure $1 \mathrm{~b}$ ).

- All the non-conform triangles in the mesh are subdivided in two by his longest edge (see figure 1c).

The last step is repeated until the mesh is completely conform (see figure 1d)
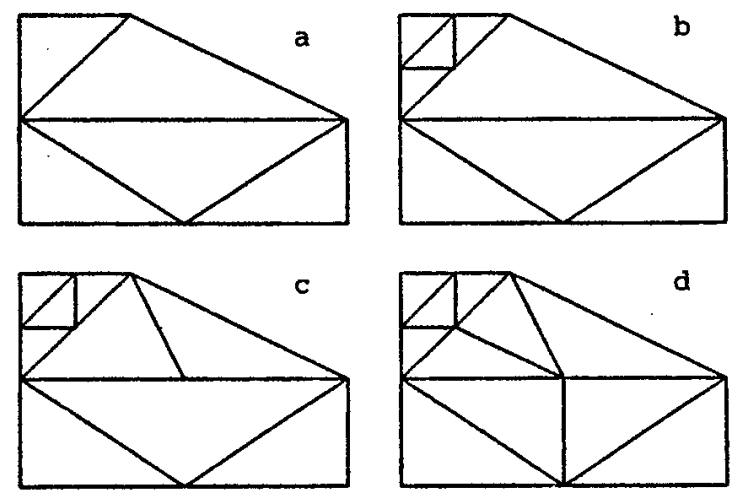

Figure 1: Densification algorithm

To use this algorithm in an adaptive environment it is necessary first to guarantee that the new elements generated will not degenerate. In $2 \mathrm{D}$ it is easy to show that the interior angle of all triangles generated by this procedure are greater or equal to the half of the smallest angle of the initial triangulation. Then, if the initial mesh has good elements, the new meshes generated will 
also have. In $3 \mathrm{D}$ there is not a similar condition, but is possible to say that the new elements will not collapse to a zero or negative volume.

\section{THE ERROR ESTIMATOR}

In this section we present two results: the error equations and the equivalence between the error and the estimator. The first result is a consequence of the integrating by parts and the second result is an easy extension of standard theorems [1] , [5] . Let us call $\Gamma$ the union of all edges of the triangulation $\mathcal{T}_{h}$ and $\Gamma_{\text {int }}=\Gamma-\partial \Omega$ the union of all the interior edges . For each $l \subset \Gamma_{\text {int }}$ let us choose an arbitrary normal direction $\mathbf{n}$ and denote the two triangles sharing this edge $T_{i n}$ and $T_{\text {out }}$, where the normal $\mathbf{n}$ points outwards of $T_{i n}$. Let

$$
\left[\left[k \frac{\partial u_{h}}{\partial n}\right]_{\ell}=\left[\nabla\left(\left.k u_{h}\right|_{T_{\text {out }}}\right)-\nabla\left(\left.k u_{h}\right|_{T_{\text {in }}}\right)\right] \cdot \mathbf{n}\right.
$$

denote the jump of $k \frac{\partial u_{k}}{\partial n}$ across the edge $l$, this value is independent of the choice of $\mathbf{n}$.

Now, we can establish the following error equations :

LEMMA : The error $e=u-u_{h}$ satisfies $\forall v \in V_{0}$ the following equation :

$+a(e, v)=\left[\sum_{K} \int_{K} R_{K} v+\int_{\partial \Omega_{2}}\left(\gamma-k \frac{\partial u_{h}}{\partial n} v\right)+\int_{\partial \Omega_{3}}\left(\beta-\alpha u_{h}-k \frac{\partial u_{h}}{\partial n} v\right)\right]+\sum_{l \in \Gamma_{i n t}} \int_{l}\left[\left[k \frac{\partial u_{h}}{\partial n}\right]_{\ell} v\right.$

where $R_{K}=\left.\left[f+\operatorname{div}\left(k \nabla\left(\left.u_{h}\right|_{K}\right)\right)-b u_{h}\right]\right|_{K}$ is the residual on the triangle $\mathrm{K}$.

For any triangle $T \in \mathcal{T}_{h}$, let $E_{T}$ be the set of its three edges, and let $\eta_{r}$ be the following indicator:

$$
\eta_{T}=\left[|T|\left\|R_{T}\right\|_{0, T}^{2}+\sum_{l \in E_{T}}|l|\left\|J_{l}\right\|_{0, l}^{2}\right]^{\frac{1}{2}}
$$

where $|T|$ and $|l|$ are the area of $T$ and the length of an edge 1 , resp. , and where

$$
J_{l}= \begin{cases}{\left[\left[k \frac{\partial u_{h}}{\partial n}\right]\right]_{l}} & \text { if } l \subset \Gamma_{i n t} \\ 0 & \text { if } l \subset \Gamma_{1} \\ 2\left[\gamma-k \frac{\partial u_{h}}{\partial n}\right]_{l} & \text { if } l \subset \Gamma_{2} \\ 2\left[\beta-\alpha u_{h}-k \frac{\partial u_{h}}{\partial n}\right]_{l} & \text { if } l \subset \Gamma_{3}\end{cases}
$$

THEOREM : Let

$$
\epsilon=\left(\sum_{T} \eta_{T}^{2}\right)^{\frac{1}{2}}
$$

be the error estimator. There are two constants $c_{0}, c_{1}$, which only depend on $\Omega$ and on the smallest angle in the triangulation $\mathcal{T}_{h}$, such that the estimates:

$$
c_{0} \epsilon \leq\left|e_{h}\right|_{1} \leq c_{1} \epsilon
$$

holds. 
a

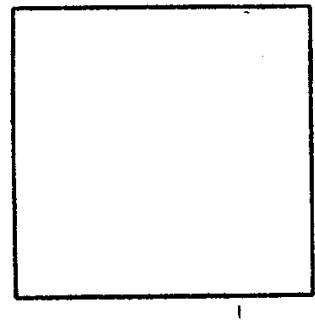

d

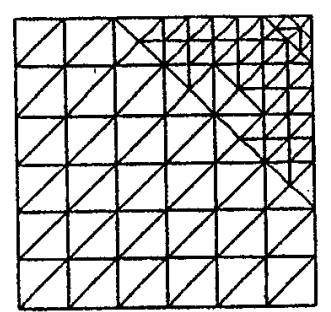

b

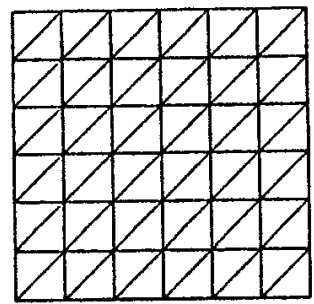

e

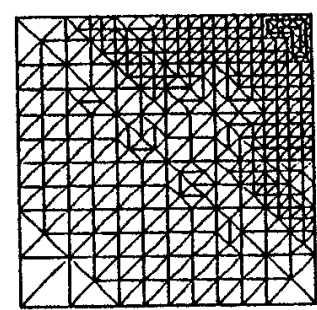

c
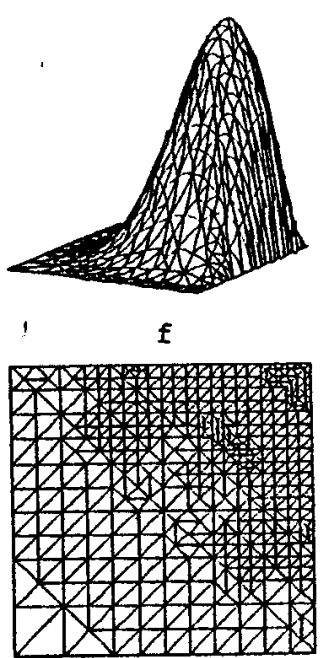

Figure 2: Solution and meshes obtained for the regular problem

\section{BEHAVIOR OF THE ERROR TERMS}

In this section we studied with some numerical examples the behavior of the two error terms, in order to know if it is possible to neglect the jumps term as suggested in [3]. We solve three diffusion problems, one with a regular solution and two with a singular solution.

Regular case: Solve the diffusion problem 1 in the region showed in figure $2 \mathrm{a}$, with coefficients

$k(x, y)=1.0$

$b(x, y)=0.0$

$f(x, y)=-6 x y\left[\left(x^{2}+y^{2}\right)(2 x y-x-y+1)-(x+y) x y\right]$

and zero Dirichlet boundary conditions in $\Gamma$. The exact solution to this problem is the smooth function

$u(x, y)=(x-1)(y-1) x^{3} y^{3}$.

Figure $2 \mathrm{~b}$ show the initial mesh and $2 \mathrm{~d}, 2 \mathrm{e}, 2 \mathrm{f}$ show the progressive adaptation to the solution using the error indicator $\eta_{T}$. In figure $2 \mathrm{c}$ we show the solution of this problem for the last mesh.

In figure 3 we show the variation of the global errors along the adaptive process. It can be seen that the jumps term is negligible, and the residual is dominant for all meshes. This agrees with the same result obtained in [3] for quadrilateral elements.

Singular case 1: Solve the diffusion problem 1 in the region showed in figure $4 \mathrm{a}$, with coefficients $k(x, y)=1.0$ 


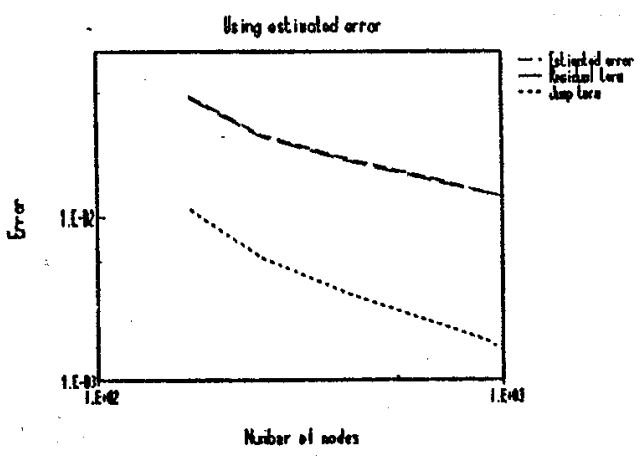

Figure 3: Error behavior for the regular problem

$b(x, y)=f(x, y)=0.0$

and with boundary conditions:

$\frac{\partial u}{\partial n}=\frac{1}{4} \cos \left(\frac{\theta}{4}\right)$ on $\Gamma_{1} u=0$ on $\Gamma_{2} \frac{\partial u}{\partial n}=0$ on $\Gamma_{3}$.

The exact solution to this problem is:

$u=r^{\frac{1}{4}} \sin \left(\frac{\theta}{4}\right)$

Observe that $u \in H^{\frac{5}{4} \epsilon}(\Omega)$ for any $\epsilon>0$ but not for $\epsilon=0$.

Figure $4 \mathrm{~b}$ show the initial mesh and $4 \mathrm{~d}, 4 \mathrm{e}, 4 \mathrm{f}$ show the progressive adaptation to the solution using the error indicator $\eta_{T}$. In figure $4 \mathrm{c}$ we show the solution of this problem for the last mesh.

In figure $5 \mathrm{a}$ we show the variation of the global errors (residual, jumps, whole estimator, and true error) along the adaptive process when the whole estimator is used. The rate of convergence is the expected, and the true error is lower than the estimated error like was predicted. It is also possible to see that the jumps term is always lower than the residual' term but not negligible. In figure $5 \mathrm{~b}$ we show what happen with the global errors when the jumps term is neglected, and the adaptive process is carried out only with the residual term. It is possible to see that the rate of convergence is deteriorated.

The last result motivate us to study another problem with a singular solution. We choose a classical one illustrated in figure 6 .

Singular case 2: Solve the diffusion problem 1 in the region showed in figure $6 a$, with coefficients

$k(x, y)=1.0$

$b(x, y)=f(x, y)=0.0$

and with boundary conditions:

$u=0$ in $\Gamma_{1} u=1$ in $\Gamma_{2} \frac{\partial u}{\partial n}=0$ in $\Gamma_{3}$

Figure $6 \mathrm{~b}$ show the initial mesh and $6 \mathrm{~d}, 6 \mathrm{e}, 6 \mathrm{f}$ show the progressive adaptation to the solution 


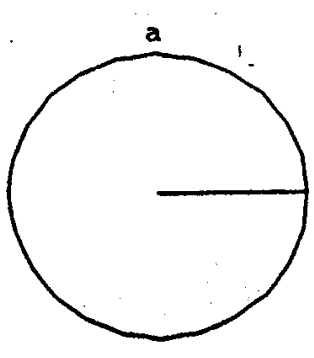

$\mathrm{d}$

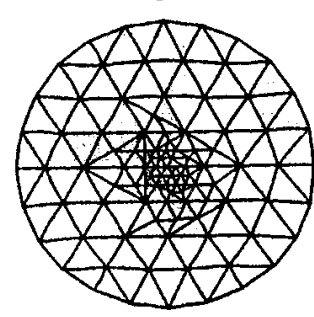

b

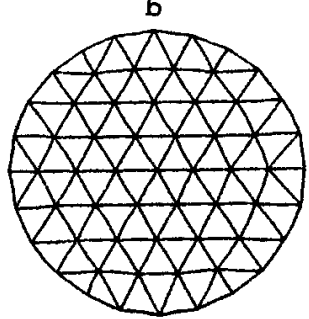

e

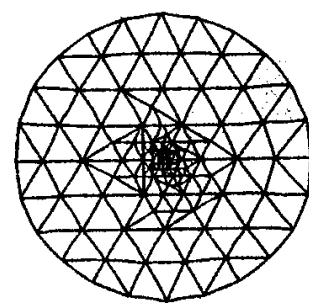

c

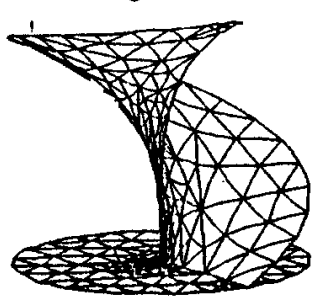

f

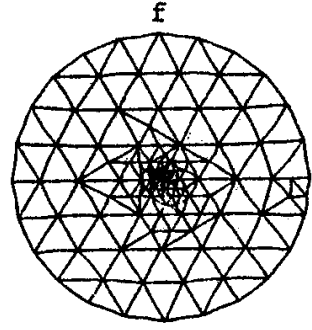

Figure 4: Solution and meshes obtained for the singular problem 1
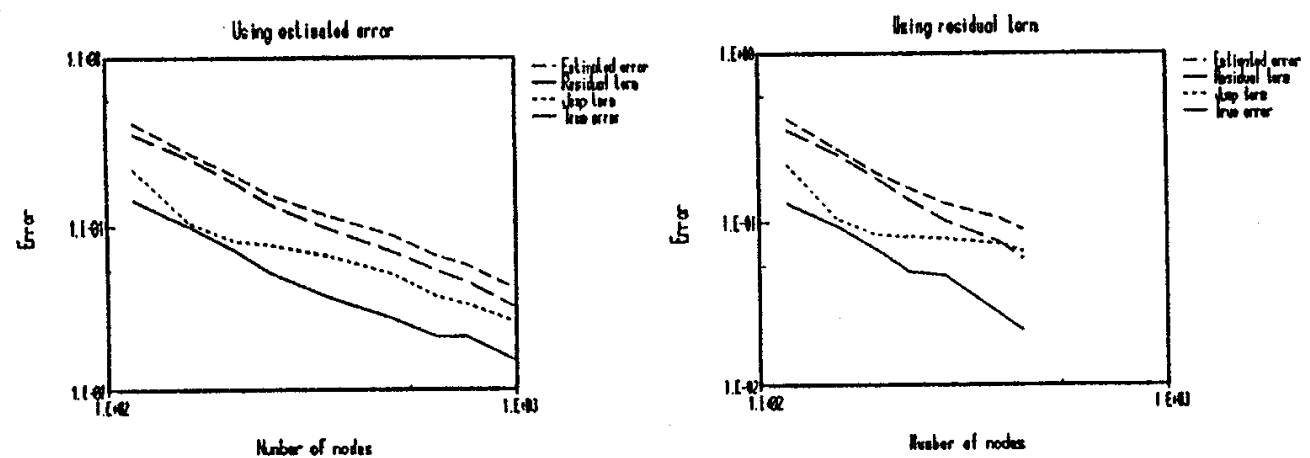

Figure 5: Error behavior for the singular problem 1

using the error indicator $\eta_{T}$. In figure $6 \mathrm{c}$ we show the solution of this problem for the last mesh.

In figures $7 \mathrm{a}$ and $7 \mathrm{~b}$ we show the variation of the global errors for this problem using the whole estimator or neglecting the jumps term. It is possible to see that in the first case, the expected rate of convergence is obtained, but not in the second. We found this is caused by the difference in the error distribution when the whole estimator or just the residual term is used. This can be observed in the figure 8 where the meshes obtained after six densification processes with these two indicators are shown. 

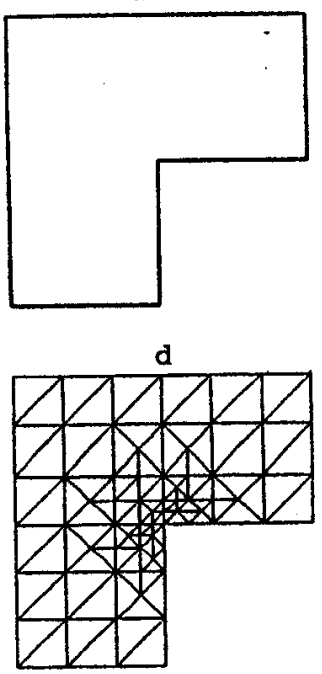

b

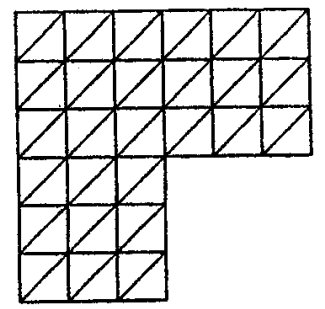

e

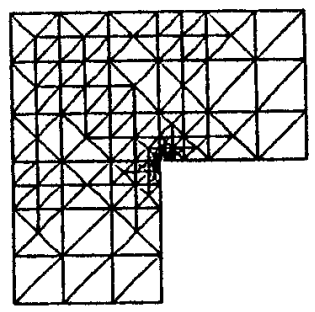

c

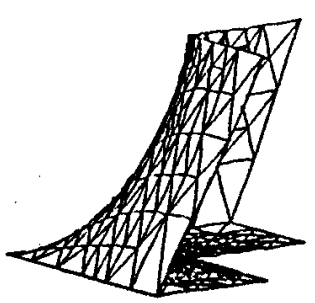

$\mathbf{f}$

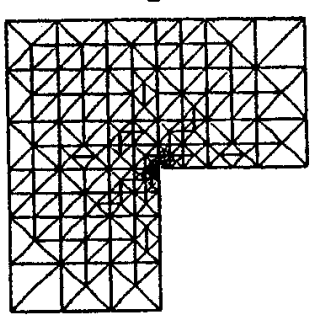

Figure 6: Solution and meshes obtained for the singular problem 2

a. 1

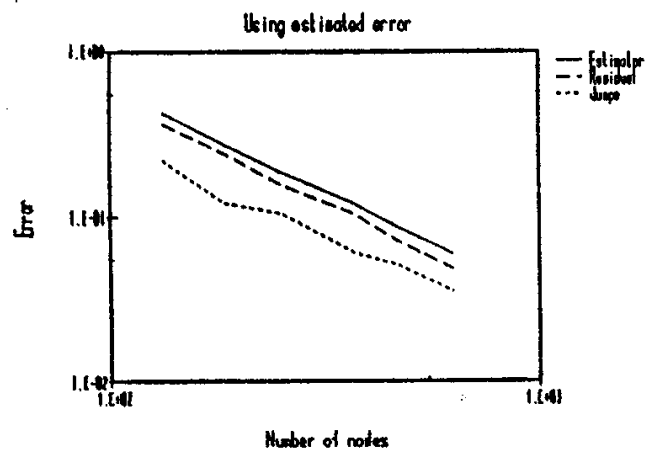

b

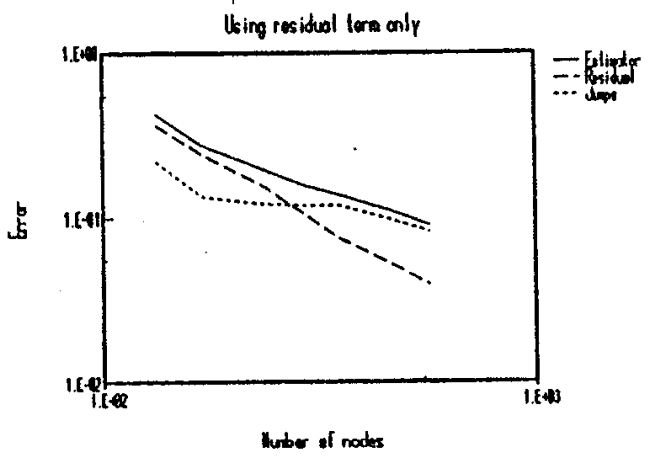

Figure 7: Error behavior for the singular problem 2

\section{SUMMARY}

The results of the previous section invalidate the assumption that the jump term of the error estimator can be neglected for quadratic elements when the optimal rate of convergence is desired, specially because the problems of practical interest in general have singular solutions.

The refinement strategy and error estimator combined with an automatic setting of boundary conditions, allows us to construct a robust and practical software to solve diffusion problems in 2D. The control over the error and the savings in preparing data make it an interesting tool for 


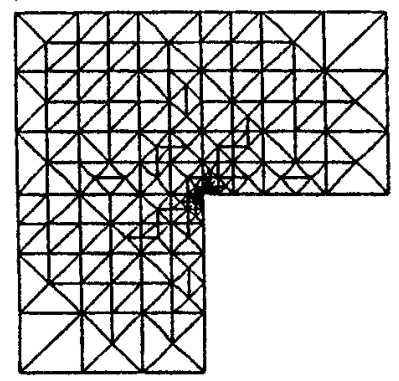

b

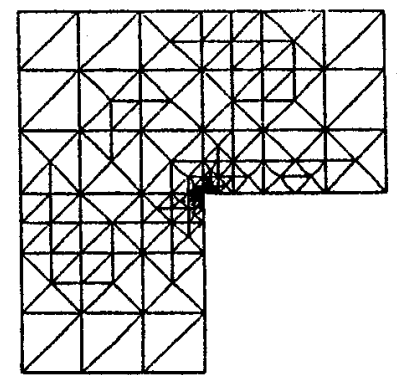

Figure 8: Meshes obtained using the whole estimator o just the residual

the engineering.

The generalization to three dimensions is possible but not straight forward, specially the densifcation algorithm. We have in progress a development in that direction.

\section{References}

[1] I. Babuška, R. Durán, R. Rodríguez ,"Analysis of the efficiency of an a posteriori error estimator for linear triangular finite elements", to appear.

[2] Babuška, I. \& Miller A. "A posteriori error estimates and adaptive techniques for the Finite Element Method", Institute for Physical Science and Technology, Technical Note BN-968 Univ. of Maryland (1981).

[3] Babuška, I. Yu D., "Asymptotically exact a posteriori error estimator for biquadratic elements ", Institute for Physical Science and Technology, Technical Note BN-1050 Univ. of Maryland (1986).

[4] Ciarlet P., "The Finite Element Method for Elliptic Problems", North Holland (1978)

[5] R. Verfürth, “A posteriori error estimators for the Stokes equations ", Numer. Math. 55, 1989, pp. 309-325.

[6] O.C. Zienkiewicz, J.Z. Zhu, " A simple error estimator and adaptive procedure for practical engineering analysis", Int. J. for Num. Methods in Eng. 24, 1987, pp. 337-357.

[7] M.C. Rivara, " A grid generator based on 4-triangles conforming mesh-refinement algorithms ", Int. J. for Num. Methods in Eng. 24, 1987, pp. 1343-1354. 
